\title{
Gouverneur's syndrome in a patient with abdominal pain: mind Crohn's disease!
}

\section{SíndROME de Gouverneur em um PACIENTE COM dOR ABdominal: CUIDAdO COM A DOENÇA DE CROHN!}

\author{
Julio Maria Fonseca Chebli ${ }^{1 *}$, Andre Avarese Figueiredo ${ }^{2}$, Pedro Duarte Gaburri $^{3}$
}

${ }^{1}$ Inflammatory Bowel Disease Center, Juiz de Fora, MG, Brazil

${ }^{2}$ Teaching Hospital of the Federal University of Juiz de Fora, Juiz de Fora, MG, Brazil

${ }^{3}$ School of Medicine, University of Juiz de Fora, Minas Gerais, MG, Brazil

Study conducted at the Federal University of Juiz de Fora, Juiz de Fora, MG, Brazil
*Correspondence:
Rua Maria José Leal , 296
\[ \begin{array}{r}\text { Juiz de Fora, MG, Brazil - ZIP Code 36036-247 } \\ \text { Phone / Fax: +55 } 323216-7122 \\ \text { chebli@globo.com }\end{array} \]
http://dx.doi.org/10.1590/1806-9282.60.03.005

Conflict of interest: none
Gouverneur's syndrome, which describes suprapubic pain, frequency, dysuria and tenesmus, is the hallmark of enterovesical fistula. ${ }^{1}$ However, Gouverneur's syndrome as the initial presentation of Crohn's disease (CD) has not been previously reported in literature.

A 26 year old man was admitted in January 2013 complaining of suprapubic pain, urinary frequency, dysuria and tenesmus over the past 3 weeks. He also reported colic abdominal pain during the past 4 months. The physical examination yielded discrete anemia. A diagnostic hypothesis of Gouverneur's syndrome related to enterovesical fistula was considered. Urine analysis showed pyuria and significant bacteriuria, and urine culture showed Escherichia coli. Contrast-enhanced computed tomography of the abdomen revealed the presence of gas in the bladder (Figure 1, white arrow). Cystoscopy revealed a localized area of erythema, edema and congestion on the bladder dome.

Skip lesions, cobblestone mucosa and ulcerations with clear margins surrounded by normal mucosa, suggestive of Crohn's colitis, were visualized at left colon on ileocolonoscopy. Endoscopic biopsies displayed lymphoplasmacytic infiltration with submucosal involvement, in addition to fissuring ulcerations in the sigmoid colon. Surgical management involved left colectomy with colocolonic anastomosis and closing primary of the bladder defect using absorbable suture. The patient had an uneventful postoperative course. One month later he was started on induction therapy with infliximab followed by maintenance with $5 \mathrm{mg} / \mathrm{kg}$ every 8 weeks. The patient remained asymptomatic 6 months later.

Crohn's disease accounts for $10 \%$ of enterovesical fistula and is the commonest cause of ileovesical fistulae. ${ }^{1}$ However, colovesical fistula is a rare condition in CD. ${ }^{2}$ Although the underlying illness is usually of intestinal origin, the majority of patients with enterovesical fistula are referred because of urinary symptoms, since the flow through the fistula predominantly occurs from the bowel to the bladder due to high compliance of the bladder and low intravesical pressure. ${ }^{2}$ The cardinal feature of enterovesical fistulae may be described as Gouverneur's syndrome. ${ }^{3}$ Other findings include abnormal urinalysis findings, pneumaturia, fecaluria, and recurrent urinary tract infections. Preoperative imaging should characterize the anatomical abnormality and exclude underlying malignancy, as well as point out the fistulous track. Computerized tomography is an excellent image modality for diagnosing enterovesical fistula. Cystoscopy is a crucial method of the investigation as it can suggest the presence of a fistula and also be useful in excluding the rare case of urological neoplasm. ${ }^{1}$ Colonoscopy is not accurate in detecting fistulas, but it is helpful in establishing the nature of the underlying bowel disease. Successful surgical management of most enterovesical fistulas from CD requires only resection of the diseased bowel, with minimal need for repair of the bladder side of the fistula. ${ }^{3}$ 


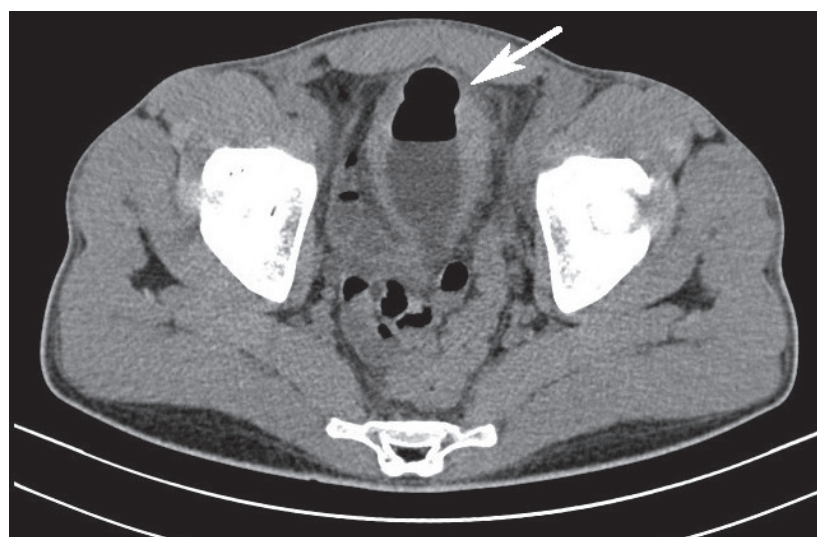

\section{References}

1. Scozzari G, Arezzo A, Morino M. Enterovesical fistulas: diagnosis and management. Tech Coloproctol 2010;14:293-300.

2. Gruner JS, Sehon JK, Johnson LW. Diagnosis and management of enterovesical fistulas in patients with Crohn's disease. Am Surg 2002; 68:714-9.

3. Garcea G, Majid I, Sutton CD, Pattenden CJ, Thomas WM. Diagnosis and management of colovesical fistulae; six-year experience of 90 consecutive cases. Colorectal Dis 2006; 8:347-52.

FIGURE 1 Contrast-enhanced computed tomography of the

abdomen showing the presence of gas in the bladder (white arrow). 